\title{
Self-Q-switched Cr:LiCAF laser
}

\author{
Ersen Beyatli, ${ }^{1}$ Alphan Sennaroglu, ${ }^{1}$ and Umit Demirbas ${ }^{2, *}$ \\ ${ }^{1}$ Laser Research Laboratory, Departments of Physics and Electrical-Electronics Engineering, \\ Koç University, Rumelifeneri, Sariyer, Istanbul 34450, Turkey \\ ${ }^{2}$ Laser Technology Laboratory, Department of Electrical and Electronics Engineering, \\ Antalya International University, Dosemealti, Antalya 07190, Turkey \\ *Corresponding author: umit79@alum.mit.edu
}

Received November 20, 2012; revised February 1, 2013; accepted February 2, 2013; posted February 4, 2013 (Doc. ID 180198); published March 14, 2013

\begin{abstract}
We report self- $Q$-switched operation of a Cr:LiCAF laser for the first time to our knowledge. Self- $Q$-switching (SQS) refers to the generation of a periodic train of $Q$-switched pulses from a laser cavity containing only the gain medium. Since SQS does not require any additional elements such as saturable absorbers or active modulators, it is far simpler and lower cost in comparison with other $Q$-switching methods. In the experiments, SQS operation was observed by using an $x$-shaped, astigmatically compensated laser cavity which contained only the Cr:LiCAF gain medium. A $140 \mathrm{~mW}$, single-mode continuous wave (cw) diode at $660 \mathrm{~nm}$ was used as the pump source. In typical cw operation, the Cr:LiCAF laser produced output powers as high as $50 \mathrm{~mW}$ with about $50 \%$ slope efficiency. The laser had a diffraction-limited output and had a spectral width of about $0.5 \mathrm{~nm}$ near $795 \mathrm{~nm}$. SQS operation could be initiated by fine tuning of the separation between the curved mirrors of the cavity and occurred at several discrete separations of the curved mirrors within the stability range of the resonator. Pulsed pumping of the pump diode, active cooling of the gain medium, and/or misalignment of the cavity end mirrors was not necessary to initiate SQS operation. In the SQS regime, the Cr:LiCAF laser produced about $5 \mu$ s wide pulses at repetition rates between 10 and $30 \mathrm{kHz}$. The corresponding pulse energies and peak powers were as high as $3.75 \mu \mathrm{J}$ and $590 \mathrm{~mW}$, respectively. SQS operation was further accompanied with (i) a decrease in the output power to the $30-45 \mathrm{~mW}$ range, (ii) an increase of the spectral bandwidth up to $10 \mathrm{~nm}$ (full width at halfmaximum), and (iii) a switching of the laser output from pure $\mathrm{TEM}_{00}$ to a structured beam containing higher-order spatial modes. We present detailed experimental data describing the temporal, spectral, and spatial characteristics of the SQS Cr:LiCAF laser, as well as the effect of curved mirror separation on SQS. The power-dependent repetition rate data were further analyzed to estimate the effective small-signal loss coefficient of the saturable absorber action. (C) 2013 Optical Society of America
\end{abstract}

OCIS codes: $\quad 140.3580,140.3540,140.3480,140.3538,160.3380,160.4760$

\section{INTRODUCTION}

Applications such as material processing, range finding, remote sensing, and nonlinear frequency conversion require lasers with pulsewidths in the nanosecond to microsecond range [1]. For obtaining short, high-energy pulses with high peak powers, $Q$-switching or gain switching techniques can be used. While the intracavity loss of a laser resonator is modulated to produce pulsed output during $Q$-switching, gain-switched operation is typically obtained via excitation of the gain medium with another pulsed laser. During $Q$-switched operation, resonator losses can be switched by using active or passive modulation schemes [2,3]. For example, acousto-optic modulators, electro-optic modulators, or rotating mirrors are typically used to alter the cavity $Q$-factor in actively $Q$-switched lasers. However, laser architectures containing such elements suffer from higher cost, lack of compactness, and increased complexity. In passively $Q$-switched lasers, the cavity losses are modulated with saturable absorbers, such as dye-based absorbers, $\mathrm{Cr}^{4+}$ :YAG (for $1 \mu \mathrm{m}$ lasers) [4], $\mathrm{Cr}^{2+}: \mathrm{ZnSe}$ (for 1.5 and $2 \mu \mathrm{m}$ lasers) [5,6], or SESAM/ SBR-based nonlinear mirrors (semiconductor saturable absorber mirror [7]/saturable Bragg reflector [8]). Compared to actively $Q$-switched lasers, passive $Q$-switching, in general, enables the construction of lower-cost and compact laser cavities. However, in passive $Q$-switching, parameters such as pulse repetition rate, pulse shape, pulse energy, and peak power are harder to independently control.

Another mechanism for the generation of nanosecond-tomicrosecond pulses is self- $Q$-switching (SQS), first named by Freund in 1968 [9]. In this technique, no special modulation elements are required inside the laser cavity to initiate and sustain the pulsing mechanism. The laser cavity simply consists of an optical resonator, a suitable gain medium, and a pump source. SQS was first observed in flashlamp-pumped ruby lasers in 1968 [9-12]. In these studies, to initiate SQS, either the laser crystal was operated at liquid nitrogen temperatures $[\underline{11}, \underline{12}]$, or one of the laser cavity end mirrors was misaligned in systems operated at room temperature $[9,10]$. We note here that in these initial studies, the pump sources were also pulsed [9-12]. Hence, SQS was used to switch the laser operation so that it produces a giant single highenergy laser pulse rather than several lower energy pulses with unequal energy and period (transition from random spiking to SQS operation) [9-12]. In the same year, the SQS effect was also observed in Nd: $\mathrm{SeOCl}_{2}$ liquid lasers under low feedback conditions (using high output coupling) [13]. A year later, SQS was reported in Nd:YAG laser media at $77 \mathrm{~K}$ [14]. In 1970, Mikaelyan et al. showed that SQS could also be initiated by adjusting the cavity length [15]. In 1976, SQS was demonstrated in a quasi-continuous wave (cw) pumped 
room-temperature $\mathrm{Nd}: \mathrm{YVO}_{4}$ laser at 1.06 and $1.34 \mu \mathrm{m}$ using cavity misalignment [16]. In 1978, for the first time, Szabo obtained SQS operation in a cw argon ion laser-pumped ruby laser operated at $80 \mathrm{~K}$ (earlier studies were all with pulsed pump sources [17]). Since then, SQS operation has been reported in flashlamp-pumped Cr:LiSAF lasers [18,19], Nd: $\mathrm{YVO}_{4}$ microchip lasers [20], erbium fiber lasers [21], broad area semiconductor lasers [22], $\mathrm{Nd}: \mathrm{YVO}_{4} / \mathrm{KTP}$ lasers [16], ytterbium fiber lasers [23], and thulium fiber lasers [24]. The underlying mechanism for SQS is not very well understood and it might differ depending on the physical characteristics of the gain medium and the operating regime of the laser. In the case of $\mathrm{Cr}^{+3}$-doped gain media such as ruby and Cr:LiSAF, the SQS effect is attributed to a nonlinear loss mechanism created by a time-dependent lens occurring inside the gain medium and originating from refractive index changes induced by the population inversion $[\underline{18}, \underline{19}, \underline{25}-\underline{28}]$. However, as a recent study by Godin et al. demonstrates, the origin of the nonresonant contribution to refractive index change in $\mathrm{Cr}^{+3}$-doped gain media (as well as the underlying physics behind the SQS effect) is not yet clear and requires further investigation [29].

In this paper, we report on the observation of the SQS effect in Cr:LiCAF gain media for the first time to our knowledge. In our experiments, SQS could be initiated in a standard 4-mirror, $x$-folded Cr:LiCAF laser that was pumped with a $\mathrm{cw}$ single-spatial-mode diode (SMD) at $660 \mathrm{~nm}$. No special modulation elements such as saturable absorbers or acousto-optic switches were present in the cavity. In the regular cw regime, at an incident pump power of $140 \mathrm{~mW}$, the Cr:LiCAF laser produced a diffraction-limited output beam with greater than $50 \mathrm{~mW}$ of output power. The free-running laser spectrum was centered around $795 \mathrm{~nm}$ and had a full width at half-maximum (FWHM) of about $0.5 \mathrm{~nm}$. However, by fine adjusting the separation of the cavity curved mirrors, we could initiate SQS in the Cr:LiCAF laser. At several values of curved mirror separation (not necessarily near the edge of the stability region), the laser switched from cw operation to SQS operation with an accompanying decrease in the output power. In the SQS regime, the laser generated pulses with around $5 \mu \mathrm{s}$ duration at repetition rates between 10 and $30 \mathrm{kHz}$, and with average powers ranging from 30 to $45 \mathrm{~mW}$. The corresponding pulse energies and peak powers were as high as $3.75 \mu \mathrm{J}$ and $590 \mathrm{~mW}$, respectively. In the SQS regime, the laser transverse mode switched from being single mode to a structured beam containing higher-order spatial modes, and spectral widening up to $10 \mathrm{~nm}$ (FWHM) was also observed. When the laser was forced to operate in single transverse mode with the insertion of an intracavity slit, the effect disappeared and the laser returned back to normal cw operation.

As mentioned above, similar self-pulsing phenomena have been reported earlier in several other gain media. However, to our knowledge, these systems were mostly flashlamp pumped, and sometimes required cooling of the crystal [12] or misalignment of the laser cavity end mirrors [10] to initiate the SQS effect. Moreover, for the first time to our knowledge, we present data that delineate the interesting role of the cavity geometry, in particular the cavity curved mirror separation, on self-pulsing phenomena. We believe that detailed investigation of the SQS effect is important for several reasons. First, as earlier studies have shown, this effect can be used in a favorable way to obtain high-energy microsecond pulses. Second, because this effect can be deleterious in building lasers operated in the pure cw or cw mode-locked regime, it is of paramount importance to be aware of the conditions under which it is initiated or it can be prevented. We believe that the experimental data presented here will help in better understanding of the underlying physical mechanisms behind the self-pulsing phenomena.

The paper is organized as follows: Section $\underline{2}$ describes the experimental setup. In Section 3, we present the experimental results on the observed SQS phenomena in detail. Finally, in Section 4 , we summarize the results, provide a general review of the $S \bar{Q} S$ process, and propose some possible causes of the effect. We note here that the aim of this paper is to present the empirical discovery in as much detail as possible. Hence, a full description and understanding of the SQS mechanism is beyond the scope of the present study.

\section{EXPERIMENTAL SETUP}

Figure 1 shows a schematic of the Cr:LiCAF laser cavity that was used in the experiments. A linearly polarized, $660 \mathrm{~nm}$, $140 \mathrm{~mW}$, AlGaInP multiquantum well, single-SMD laser (HL6545MG, Hitachi) with a diffraction-limited beam profile was used as the pump source. An aspheric lens with a focal length of $4.5 \mathrm{~mm}(\mathrm{NA}=0.55)$ collimated the diode output beam. A converging lens with a focal length of $60 \mathrm{~mm}$ focused the collimated pump beam into the Cr:LiCAF crystal. The Cr:LiCAF laser resonator was a standard $x$-folded, astigmatically compensated cavity consisting of two curved pump mirrors, each with a radius of curvature (roc) of $75 \mathrm{~mm}$ (M1 and M2), a flat end high reflector (M3), and a flat output coupler (OC). Mirrors M1-M3 had a reflectivity of greater than $99.9 \%$ in the $730-910 \mathrm{~nm}$ wavelength range and had $>98 \%$ transmission in the pumping window. The cavity arm lengths were $25 \mathrm{~cm}$ (OC arm) and $35 \mathrm{~cm}$ (M3 arm), giving a laser mode size of $\sim 15-20 \mu \mathrm{m}$ inside the Cr:LiCAF crystal. The gain material was a $4 \mathrm{~mm}$ long, $2.6 \mathrm{~mm}$ wide, and $1 \mathrm{~mm}$ thick piece of Cr:LiCAF crystal with $5 \mathrm{~mol}$. \% chromium (corresponding chromium concentration $\sim 4.75 \times 10^{20}$ ions $/ \mathrm{cm}^{3}$ [30]) and was grown at the Leibniz Institute for Crystal Growth by using the Czochralski method [31-33]. The crystal absorbed 95\% of the TM polarized pump light at $660 \mathrm{~nm}$. The estimated passive losses of the Cr:LiCAF crystal were below $0.15 \%$ per $\mathrm{cm}$, which corresponds to a figure of merit of above 2000 [34]. Neither the pump diode nor the Cr:LiCAF laser crystal required active cooling. Temporal characterization of the laser was performed using a $1 \mathrm{GHz}$ Si photodetector and a $500 \mathrm{MHz}$

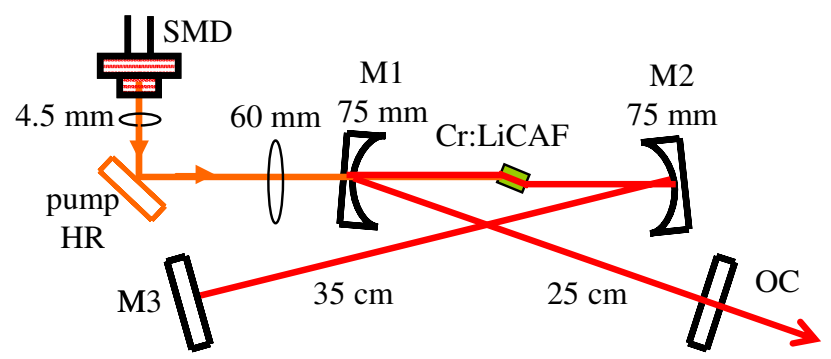

Fig. 1. (Color online) Schematic of the Cr:LiCAF laser pumped with one single-spatial-mode diode (SMD) laser. The laser operates in SQS mode at some discrete values of the curved mirror (M1-M2) separation. 


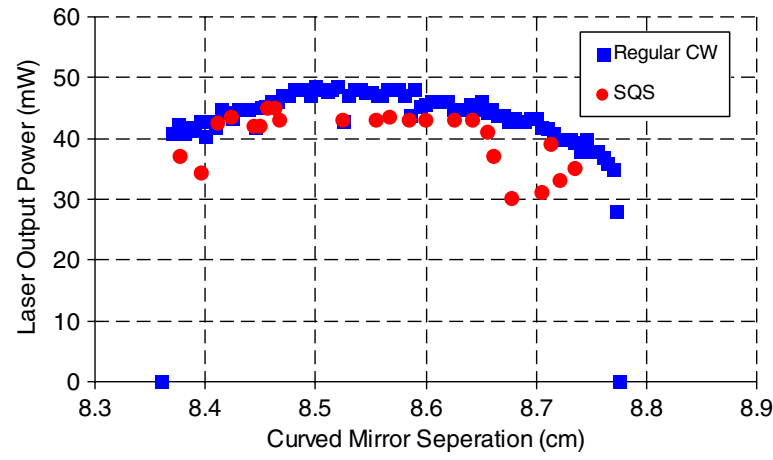

Fig. 2. (Color online) Experimentally measured variation of the laser output power as a function of the curved mirror separation within the outer stability range. Squares, pure cw operation; Circles, SQS operation.

digital sampling oscilloscope using a $10 \mathrm{k} \Omega$ termination. The laser spectrum was measured with a scanning, fiber-coupled spectrometer (Ocean Optics USB2000) and the laser transverse mode was monitored with the Coherent Lasercam HR beam profiler.

\section{EXPERIMENTAL RESULTS}

In our experiments, we observed the SQS operation of the Cr: LiCAF laser in a reproducible manner at several positions within the cavity stability range. To elucidate this regime of operation, the power efficiency data shown in Fig. $\underline{2}$ were taken. The graph shows the measured variation of the laser output power within the outer stability range as a function of the curved mirror separation (M1-M2 separation in Fig. 1). The data were taken at an incident pump power of about $140 \mathrm{~mW}$ by using a $0.75 \%$ transmitting output coupler and without any active cooling of the gain medium. Also, while taking the data, the position of the laser crystal, pump focusing lens, and the cavity alignment were first optimized at the center of the stability range. Then, during the measurements, only the curved mirror separation was varied without adjusting any other cavity component.

At most values of the curved mirror separation, the Cr:LiCAF laser operated in regular cw regime (shown with solid squares in Fig. 2). At these positions, the laser was quite stable with a $\mathrm{TEM}_{00}$ output $\left(\mathrm{M}^{2}\right.$ was below 1.05) and average powers of $40-50 \mathrm{~mW}$ could be obtained. Furthermore, the laser output was quite stable with no noticeable tendency toward $Q$-switching and typical free-running laser line widths were about $0.5 \mathrm{~nm}$ near the center wavelength of $795 \mathrm{~nm}$. However, at several distinct positions within the stability range (marked by solid circles in Fig. 2), the laser switched to SQS operation. Once initiated, SQS operation was quite stable and could be reproduced at these points in a repeatable fashion. We note here that obtaining SQS operation required fine adjustment of the curved mirror separation within about $10 \mu \mathrm{m}$. At these specific curved mirror distances, the laser output power was in general lower compared to the cw case. Moreover, during SQS operation, the laser transverse mode also switched from being single mode to a structured multimode, and spectral widening was also observed. However, during SQS operation, when we inserted a mechanical slit inside the laser resonator to force the laser to operate in pure $\mathrm{TEM}_{00}$ mode, the laser switched back to pure cw operation. This was accompanied by an increase in the output power and a decrease in the spectral bandwidth.

In summary, with our setup, transition to SQS regime of operation was signaled by several factors including (i) an abrupt decrease in the output power, (ii) a sudden widening of the output optical spectrum, (iii) pulsing of the laser output in the time domain, and (iv) a change in the transverse mode structure. We also note here that the SQS positions indicated in Fig. 2 with solid circles do not represent the complete list as there were other positions, not marked in Fig. 2, where weak SQS tendency was observed. Moreover, even though SQS operation could be initiated at several different positions within the stability range, the temporal characteristics of the laser output, such as pulse shape, pulse modulation depth, and repetition frequency varied at different positions. We have also checked the SQS operation tendency within the inner stability range. The inner stability range showed similar SQS operation parameters. However, compared to the outer stability range, we observed SQS operation at fewer discrete positions. In the remaining parts of this section, we will provide representative data (Figs. 3-7) to describe the rich behavior of the SQS operation that was obtained at different curved mirror separation distances.

Figure 3 shows the output characteristics at the curved mirror separation of $8.45 \mathrm{~cm}$ taken at an incident pump power of $140 \mathrm{~mW}$. For this case and similar others, we have observed relatively weak widening of the optical spectrum (widening from about 0.5 to $1.2 \mathrm{~nm}$ ). When SQS operation was initiated by fine adjustment of the curved mirror separation, the average output power of the Cr:LiCAF laser decreased from 45 to $42 \mathrm{~mW}$. The laser produced $6.5 \mu \mathrm{s}$ long pulses at a repetition rate around $17 \mathrm{kHz}$ (11\% duty cycle). The corresponding pulse energy and peak power are $2.5 \mu \mathrm{J}$ and $360 \mathrm{~mW}$, respectively. The SQS operation also transferred the $\mathrm{TEM}_{00}$ laser output to a structured beam, consisting of higher-order rectangular
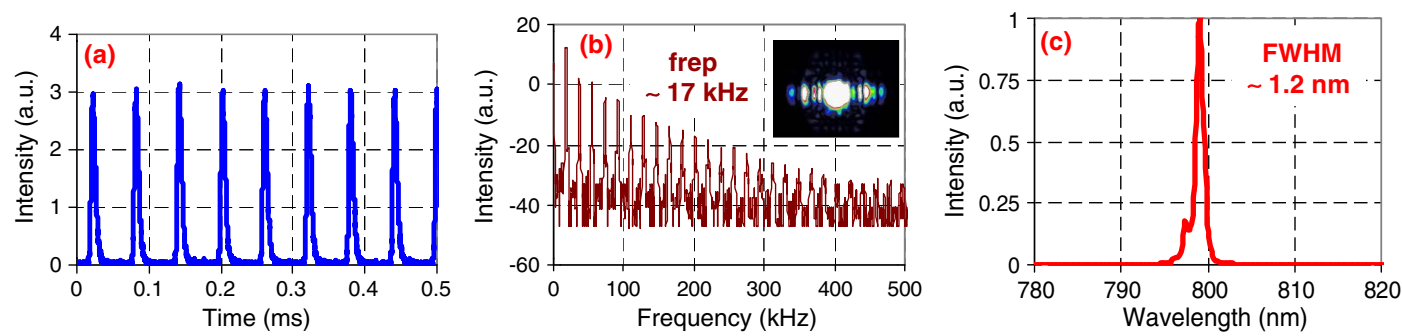

Fig. 3. (Color online) (a) Measured temporal output profile, (b) fast Fourier transform of the output, and (c) the measured optical spectrum for the SQS Cr:LiCAF laser at the curved mirror separation of $8.45 \mathrm{~cm}$. Inset figure shows the output transverse mode. The laser produced $6.5 \mu \mathrm{s}$ long pulses at a repetition rate around $17 \mathrm{kHz}$. The average output power was $42 \mathrm{~mW}$, corresponding to a pulse energy of about $2.5 \mu \mathrm{J}$ and a peak power of $380 \mathrm{~mW}$. The optical spectrum of the pulse was centered around $799 \mathrm{~nm}$ with a FWHM of $1.2 \mathrm{~nm}$. The incident pump power was $140 \mathrm{~mW}$. 

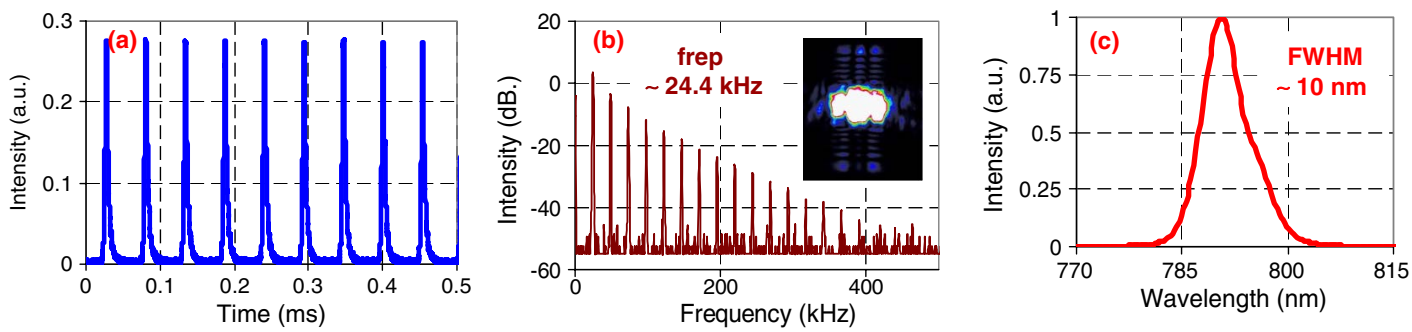

Fig. 4. (Color online) (a) Measured temporal output profile, (b) fast Fourier transform of the output, and (c) the measured optical spectrum for the SQS Cr:LiCAF laser at the curved mirror separation of $8.46 \mathrm{~cm}$. Inset figure shows the output transverse mode. The laser produced $4 \mu \mathrm{s}$ long pulses at a repetition rate around $24 \mathrm{kHz}$. The average output power was $40 \mathrm{~mW}$, corresponding to a pulse energy of about $1.6 \mu \mathrm{J}$ and a peak power of $400 \mathrm{~mW}$. The optical spectrum of the pulse was centered around $790 \mathrm{~nm}$ with a width (FWHM) of $10 \mathrm{~nm}$. The incident pump power was $140 \mathrm{~mW}$.

modes [see inset in Fig. 3(b)]. In general, during SQS operation, the laser transverse mode had rectangular transverse mode patterns. For most of the cases, the mode became multimode only along one axis and stayed as a fundamental mode along the others axis (modes such as $\mathrm{TEM}_{10}, \mathrm{TEM}_{20}, \mathrm{TEM}_{30}$, $\mathrm{TEM}_{01}, \mathrm{TEM}_{02}$, and $\mathrm{TEM}_{03}$ were observed). As an example, at the mirror separation of $8.45 \mathrm{~cm}$, the $\mathrm{M}^{2}$ parameter of the beam was measured to be around 5 along the fast axis. One should not forget that, as indicated by earlier studies, the observed transverse beam profile as well as the divergence and, hence, the $\mathrm{M}^{2}$ of the beam are time dependent during SQS operation [27]. Hence, the beam profile shown in Figs. $\underline{3}$ and $\underline{4}$ are time-averaged acquisitions. In our experiments, once the desired curved mirror separation was obtained, the SQS operation was in general quite robust, meaning that the laser stayed in the SQS regime and did not jump between SQS and regular cw operation. The pulse widths were also quite stable and the pulse to pulse energy variation was below $20 \%$. For the SQS configuration summarized in Fig. 3, the repetition frequency of the laser varied by about $20 \%$ around $17 \mathrm{kHz}$ due to fluctuations in laser dynamics [see Fig. 3(b)]. It may be possible to further reduce the jitter by active modulation of the pump power or by quasi-cw pumping.

Even more stable SQS operation with lower amount of variation in the laser parameters (10\%) was obtained at several other curved mirror separations. For this case, the widening of the optical spectrum was also more pronounced. As a typical example, Fig. $\underline{4}$ shows the measured SQS laser parameters at a curved mirror separation of $8.46 \mathrm{~cm}$. The laser output spectrum was significantly broader with a width (FWHM) of $10 \mathrm{~nm}$. The coherence length of such a broad-bandwidth

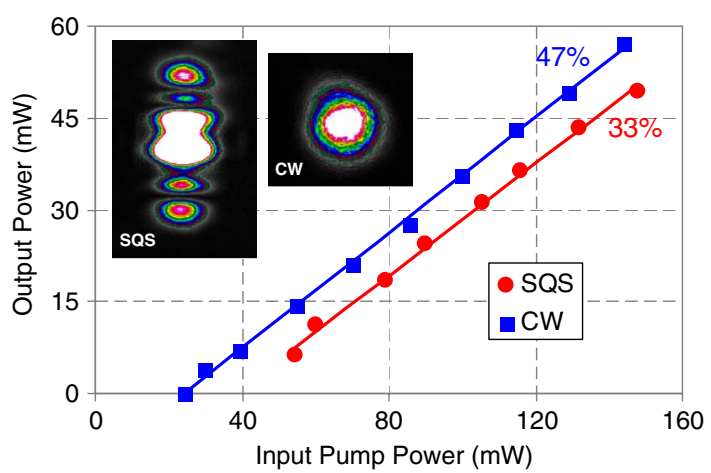

Fig. 5. (Color online) Power efficiency data of the cw and SQS Cr:LiCAF laser operated with a $0.75 \%$ output coupler. Inset figures show the typical beam profiles for each case. source around these wavelengths is about $30 \mu \mathrm{m}$ in air, which could be useful for applications such as high axial resolution optical coherence tomography [35].

Using the $0.75 \%$ output coupler, we also observed SQS operation at repetition rates as low as $12 \mathrm{kHz}$. The pulses were $6 \mu \mathrm{s}$ long and the laser average power was around $45 \mathrm{~mW}(7 \%$ duty cycle). The optical spectrum width was centered around $790 \mathrm{~nm}$ with a FWHM of $7 \mathrm{~nm}$. The corresponding pulse energy and peak power were $3.75 \mu \mathrm{J}$ and $587 \mathrm{~mW}$, respectively. These results show that, using SQS, the peak power of the laser can be scaled by about 10 times. Such a scaling can be useful for performing nonlinear experiments such as intracavity second harmonic generation [34]. We note here that we have also observed SQS in our earlier experiments with higher power multimode or tapered diode pumped Cr:LiCAF laser systems [36,37]. Hence, using high-power diodes, it is feasible to scale the pulse energies and peak powers reported in this study to at least $200 \mu \mathrm{J}$ and $20 \mathrm{~W}$ levels, respectively. On the other hand, the current system, with reasonable average and peak powers, is still attractive for many applications due to its simplicity, compactness, and low cost.

By using standard passive $Q$-switching theory, we can model the SQS action as a simple saturable absorber and use the experimental pump-dependent repetition rate data to determine the small-signal saturable loss. In the presence of a saturable absorber with a small-signal loss of $Q_{o}$ (also known as the modulation depth), the repetition frequency $f_{\text {rep }}$ of the pulse train can be determined from the equation [38-41]

$$
f_{\text {rep }}=\frac{1}{\tau_{f}} \frac{G_{o}}{2 Q_{o}},
$$

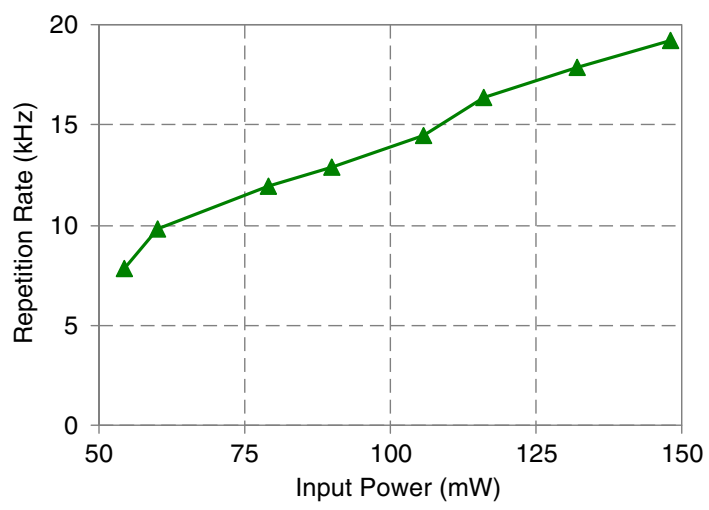

Fig. 6. (Color online) Measured variation of the frequency of the repetitive pulse train as a function of the input pump power. 


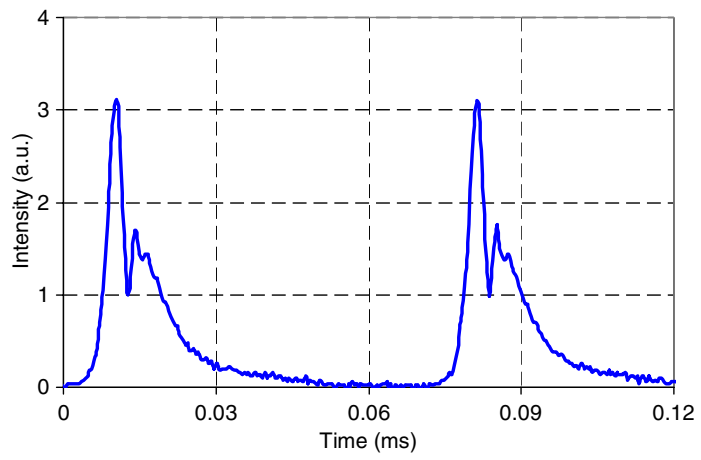

Fig. 7. (Color online) Measured temporal output profile for the SQS Cr:LiCAF laser taken with a $0.1 \%$ output coupler. The laser produced $4.8 \mu \mathrm{s}$ long pulses at a repetition rate around $15 \mathrm{kHz}$. One can also notice oscillations in the pulse intensity at higher frequencies (around $250 \mathrm{kHz}$ ), which might be caused by relaxation oscillations.

where $\tau_{f}$ is the fluorescence lifetime of the gain medium (175 $\mu \mathrm{s}$ for Cr:LiCAF) [30] and $G_{o}$ is the round-trip small-signal gain. For a four-level laser system at low pumping levels, $G_{o}$ varies approximately linearly with the pump power and can be estimated from the efficiency equation given by

$$
P_{\mathrm{out}}=T \frac{P_{\mathrm{sat}}}{2}\left(\frac{G_{o}}{G_{\mathrm{th}}}-1\right) .
$$

Above, $P_{\text {out }}$ is the laser output power and $T$ is the intensity transmission of the output coupler. $G_{\text {th }}$ is the fractional threshold gain needed to reach lasing and can be expressed as $G_{\text {th }}=(1 / 2)\left(L_{c}+T\right) . L_{c}$ is the round-trip loss of the cavity and was determined to be around $0.2 \%$ [34]. The saturation power $P_{\text {sat }}$ is further given by

$$
P_{\mathrm{sat}}=\frac{h v_{L}}{\sigma_{e} \tau_{f}} A,
$$

where $h$ is the Planck's constant, $h v_{L}$ is the laser photon energy, $\sigma_{e}$ is the emission cross section $\left(1.3 \times 10^{-20} \mathrm{~cm}^{2}\right)$, and $A$ is the effective cross-sectional area of the laser beam inside the gain medium. The SQS data that were used for the determination of $Q_{0}$ were taken at the curved mirror separation of $8.46 \mathrm{~cm}$. By using the standard ABCD analysis, the laser beam waist inside the gain medium was estimated to be $25 \mu \mathrm{m}$, giving $A=2.75 \times 10^{-5} \mathrm{~cm}^{2}$. Hence, from Eq. (3), the corresponding saturation power was determined to be about $3 \mathrm{~W}$.

To determine the small-signal round-trip gain $G_{0}$, the power efficiency data of the $\mathrm{cw}$ and SQS Cr:LiCAF laser were recorded as shown in Fig. 5. At each pump power level, the cw output power was used together with Eqs. (1) and (2) above to determine the corresponding value of $\bar{G}_{0}$. Fig. 6 shows the measured variation of the repetition rate of the pulse train as a function of the incident pump power. By using Eqs. (1) and (2), the variation of the repetition frequency $f_{\text {rep }}$ as a function of $G_{0}$ was determined. Linear least squares fit to the data gives a best-fit value of $0.5 \%$ for $Q_{0}$. Also note that when the laser made a transition to SQS regime, the threshold pump power increased from 24 to $44 \mathrm{~mW}$ due to the additional $0.5 \%$ small-signal loss represented by $Q_{0}$. Moreover, the slope efficiency also decreased from $47 \%$ to $33 \%$. For both cases, the transmission of the output coupler was $0.75 \%$.
We note in passing that SQS operation could also be attained with output couplers having different transmission. In our experiments, we observed SQS operation with $0.1 \%$, $0.25 \%, 2 \%$, and $3 \%$ transmitting output couplers as well. On the other hand, using a 5\% output coupler, which produced only $25 \mathrm{~mW}$ of output power, we have been unable to observe stable SQS operation. If the system is pumped at a higher level (for example, by pumping with 2 diodes), it should be possible to observe SQS operation with the 5\% output coupler. Moreover, with lower output coupling, SQS operation showed a threshold-like behavior and started only after the pump power reached a threshold value as shown in Fig. $\underline{6}$. Furthermore, the stability of SQS increased at higher pump power levels. All of these observations show that observation of SQS requires the laser to reach a certain intracavity power level. Here, Cr:Colquirrites have an advantage due to the possibility of growing crystals with a large figure of merit. This enables the construction of high $Q$ cavities that can store a large amount of intracavity power.

Similar to the $0.75 \%$ output coupler, the output couplers with $0.1 \%, 0.25 \%, 2 \%$, and $3 \%$ transmission also required fine adjustment of the curved mirror separation to initiate SQS operation. Similarly, SQS could be observed at several discrete curved mirror separations as in Fig. 2. Roughly speaking, with lower output coupling, SQS operation could be initiated at larger number of discrete mirror separation values. This might be due to the presence of higher intracavity powers at lower output coupling.

Since SQS parameters strongly depend on the exact mirror separation value that is used, it is not possible to make a very precise comment on the effects of output coupling on SQS parameters. However, comparing the SQS operation results taken with different output couplers, we can qualitatively conclude that with increasing output coupling, (i) SQS pulse width decreases, (ii) pulse repetition frequency increases, and (iii) the output optical spectral width decreases. For example, $2.8 \mu$ s long pulses at $27 \mathrm{kHz}$ repetition rate were obtained with an average power of $33 \mathrm{~mW}$ by using a $3 \%$ output coupler $(1.2 \mu \mathrm{J}$ pulse energy, $410 \mathrm{~mW}$ peak power, $7.6 \%$ duty cycle, and $37 \mu$ s period). The spectral width was $5.8 \mathrm{~nm}$ wide and was centered at around $780 \mathrm{~nm}$. On the other hand, $4.8 \mu$ s long pulses at $15 \mathrm{kHz}$ repetition rate were generated at an average power of $19 \mathrm{~mW}$ using the $0.1 \%$ transmitting output coupler $(1.3 \mu \mathrm{J}$ pulse energy, $250 \mathrm{~mW}$ peak power, $7.2 \%$ duty cycle, $67 \mu$ s period). For this case, the spectral width was centered at around $794 \mathrm{~nm}$ with a FWHM of about $10 \mathrm{~nm}$.

In our experiments, the repetition rate during the SQS operation varied between 10.35 and $31 \mathrm{kHz}$ at different curved mirror separation distances (at the full pump power level). Here, we would like to first compare this frequency range with the relaxation oscillation frequency of the Cr:LiCAF laser. When a laser is pumped sufficiently above the lasing threshold, and if the laser gain medium has a long upper state lifetime, the relaxation oscillation frequency can be estimated using

$$
w_{R} \approx \sqrt{\frac{r\left(L_{c}+T\right)}{\left(\tau_{f}\right)\left(T_{R}\right)}},
$$

where $r$ is the pumping parameter ( $r$ is the ratio of the pump power to the threshold pump power), and $T_{R}$ is the cavity 
round trip time [42]. Taking $T_{R}$ as $5 \mathrm{~ns}, r$ as 10, $T$ as $0.0075, L_{c}$ as 0.002 , the estimated relaxation oscillation frequency of our laser comes to $330 \mathrm{kHz}$, which is significantly above the observed range of SQS repetition frequencies. However, in some configurations in which the pulse width is relatively long, we have observed a higher-frequency oscillation in the pulse intensity. For example, Fig. 7 shows the measured temporal profile of the laser output taken with the $0.1 \%$ transmitting output coupler. The pulse train intensity had a weak oscillation around $250 \mathrm{kHz}$. We believe that the observed intensity oscillation within the pulse envelope was due to relaxation oscillations.

As was mentioned in the Introduction, most of the earlier studies report SQS operation in pulsed pumped systems. Hence, in these systems, the SQS frequency was the same as the pumping frequency. As an example, Weber et al. obtained 200-500 ns pulses from a Cr:LiSAF laser pumped by rectangular $60 \mu$ s flashlamp pulses at a repetition rate of $100 \mathrm{~Hz}$ [18]. There are only a few reports of SQS operation in cw-pumped systems. In [43], SQS has been reported in a cw-pumped $\mathrm{Nd}: \mathrm{YVO}_{4}$ laser, and pulse widths of 40 ns were obtained by misalignment of cavity end mirrors. However, the SQS repetition frequency was not mentioned in this study. In another study, SQS was obtained in a cw-pumped ruby laser, and pulses with $2 \mathrm{~ns}$ duration at $3 \mathrm{kHz}$ repetition rate were obtained [17]. In comparison, the SQS frequency we observed in Cr:LiCAF is about 5 times larger than that reported in ruby. The laser systems are quite different and the difference in the SQS frequencies may be due to numerous factors including the fluorescence lifetime, small-signal gain, and the effective small-signal loss parameter of the effective SQS action as can be seen from Eq. (1). In particular, the fluorescence lifetime in ruby ( $3 \mathrm{~ms}$ ) is about 17 times higher than that in Cr:LiCAF.

\section{SUMMARY AND DISCUSSION}

In summary, to the best of our knowledge, this study reports SQS operation of a Cr:LiCAF laser for the first time. Furthermore, this is also the first demonstration of SQS in a cw-pumped, room-temperature solid-state laser. Unlike most of the earlier studies, initiation of SQS action in the present study did not require the misalignment of the cavity optics. SQS could be initiated just by fine adjustment of the curved mirror separation within the cavity stability range. Moreover, SQS was observed at several different values of curved mirror separation and these points were not necessarily at the edge of the stability range. Finally, the pump power levels we have used here are also relatively low. Hence, compared to the earlier reports in the literature, this study shows unique characteristics of the mechanism responsible for obtaining SQS. We hope that the data presented in this work will help better the understanding of SQS and the physics behind it.

In explaining the SQS effect, earlier studies have proposed or investigated many mechanisms as possible causes, including backward Brillouin scattering [9], thermal lensing (ther-

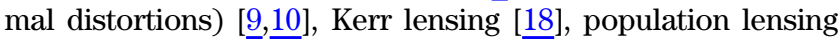
[19], dynamic realignment [10], super-radiant emission [10], saturable absorption process by partial shielding of the gain medium [12], stimulated scattering phenomena in the gain medium, and so on. Later studies have shown that the SQS effect is most probably caused by a time-dependent lens created inside the gain medium due to the nonuniform refractive index variations. This time-dependent lens, combined with a hard or soft aperture, results in intensity-dependent losses, which results in SQS operation [25]. Hence the small-signal loss parameter $Q_{0}$ that was estimated in the earlier section represents the effective loss brought about by the SQS mechanism at low intensities.

There are several physical mechanisms that can generate a time-dependent lens inside gain media via nonuniform refractive index variations. These include (i) thermal effects (thermal lensing), (ii) nonlinear Kerr effect (Kerr lensing), and (iii) nonthermal electronic effects (population lensing). Studies on flashlamp-pumped SQS Cr:LiSAF lasers have shown that, among these effects, population lensing is the most probable cause for the SQS behavior [18, $\underline{19}, \underline{25-28]}$. It is believed that this refractive index difference is due to a variation of ionic polarizability when the chromium ions change energy states [25]. This refractive index change is also nonuniform due to the Gaussian distribution of the pump beam (due to the position dependence of population inversion), and creates a time-dependent lens within the gain medium. Refractive index changes due to the variation of ionic polarizability $\left(\Delta \alpha_{p}=\left(\alpha_{\mathrm{ex}}-\alpha_{g}\right)\right.$, where $\alpha_{\mathrm{ex}}$ and $\alpha_{g}$ refers to excited and ground state polarizability) can be expressed as $[\underline{25}, \underline{44}]$ :

$\Delta n_{\mathrm{pop}}(r, t)=\frac{2 \pi}{n_{0}} f_{L}^{2} N_{T} \Delta \alpha_{p} \frac{N_{\mathrm{ex}}(r, t)}{N_{T}}=K \frac{N_{\mathrm{ex}}(r)}{N_{T}}=C_{K} N_{\mathrm{ex}}(r, t)$,

where $n_{0}$ is the linear refractive index, $f_{L}$ is the Lorentz local field correction factor, $N_{T}$ is the total density of the $\mathrm{Cr}^{3+}$ ions, $N_{\text {ex }}$ is the excited ion population density, $K$ is a single constant which can be expressed as $K=\left(2 \pi / n_{0}\right) f_{L}^{2} N_{T} \Delta \alpha_{p}$, and $C_{K}$ is a normalized proportionality constant that is useful to compare results taken with materials at different concentrations $\left(C_{K}=K / N_{T}\right)$. There are several experimental techniques on measuring the magnitude and sign of these parameters including differential and pump probe interferometry, degenerate four-wave mixing, two-wave mixing, $z$-scan, far-field intensity pattern, and divergence measurements [44]. These techniques have been used to determine the relevant parameters of ruby, Cr:LiSAF and Cr:GSGG [25-27, $\underline{29}, \underline{44}-46]$. As an example refractive index changes on the order of $10^{-6}$ has been estimated in Cr:LiSAF, which could generate time-dependent population lenses with a focal length of about $10 \mathrm{~m}$ [19].

The temporal and frequency response of the refractive index changes have been relatively well understood by now. Moreover, resonant and nonresonant contributions to material susceptibility have been identified and could be discriminated relatively well (for example, the variation of the ionic polarizability is due to the nonresonant contribution to the susceptibility) [47]. However, the spectroscopic origin of the nonresonant contribution to refractive index change is not yet fully understood and recent numerical calculations based on this model show disagreements with some of the experimental data [29]. Moreover, there are also other possible physical mechanisms that should be considered as the potential cause of population lensing effect. One of these physical mechanisms is the photoelastic effect, originating from the "elastic dilation of the $\mathrm{CrO}_{6}$ cluster in the ${ }^{4} \mathrm{~T}_{2}$ excited state of the $\mathrm{Cr}^{+3}$ ions" $[29,48,49]$. In short, further detailed research 
is required for better understanding of the SQS phenomena as well as the physical mechanism that causes the population lensing effect.

For our case, there does not exist any earlier reports of SQS in Cr:LiCAF. Hence, the physical mechanism behind the SQS effect is not yet studied. The literature also lacks studies of population lensing effect in the Cr:LiCAF gain media. However, both Cr:LiCAF and Cr:LiSAF belong to the Cr:Colquiriite family and, roughly speaking, they possess similar optical, physical, and spectroscopic properties [50]. Actually, when we repeat our SQS experiments with a Cr:LiSAF laser (just replacing the Cr:LiCAF crystal with a $5 \mathrm{~mm}$ long, $1.5 \%$ chromium-doped Cr:LiSAF), we have also observed SQS by fine adjustment of the curved mirror separation. However, SQS operation was less stable and could be obtained only at fewer locations. Hence, both media also show similarities in terms of SQS operation. Earlier studies with Cr:LiSAF gain media suggest that SQS in Cr:LiCAF is also probably due to the time-dependent population lens created by population inversion $[\underline{18}, \underline{19}, \underline{25-28}]$. However, it is hard to make concluding remarks and further studies are required for better understanding of a general theory of SQS action.

\section{ACKNOWLEDGMENTS}

We thank Stefan Eggert, Christian Beschle, and Adnan Kurt for help in the experiments, and Alfred Leitenstorfer, Reinhard Uecker, and Detlef Klimm for providing some of the optics and optomechanics that were used in this study. We acknowledge partial support from TÜBİTAK (The Scientific and Technical Research Council of Turkey, 121T220), European Union Marie Curie Career Integration Grant (PCIG11-GA-2012-321787), Alexander von HumboldtFoundation, Center for Applied Photonics of Konstanz University, and Antalya International University.

\section{REFERENCES}

1. R. Paschotta, Encyclopedia of Laser Physics and Technology (Wiley-VCH, 2008).

2. J. J. Degnan, "Optimization of passively $Q$-switched lasers," IEEE J. Quantum Electron. 31, 1890-1901 (1995).

3. K. Du, D. Li, H. Zhang, P. Shi, X. Wei, and R. Diart, "Electrooptically $Q$-switched NdYVO 4 slab laser with a high repetition rate and a short pulse width," Opt. Lett. 28, 87-89 (2003).

4. A. Sennaroglu, "Broadly tunable $\mathrm{Cr}^{4+}$ doped solid-state lasers in the near infrared and visible," Prog. Quantum Electron. 26, 287-352 (2002).

5. A. V. Podlipensky, V. G. Shcherbitsky, N. V. Kuleshov, V. P. Mikhailov, V. I. Levchenko, and V. N. Yakimovich, " $\mathrm{Cr}^{2+}: \mathrm{ZnSe}$ and $\mathrm{Co}^{2+}: \mathrm{ZnSe}$ saturable-absorber $Q$ switches for $1.54 \mu \mathrm{m}$ Er:glass lasers," Opt. Lett. 24, 960-962 (1999).

6. H. Cankaya, U. Demirbas, A. K. Erdamar, and A. Sennaroglu, "Absorption saturation analysis of $\mathrm{Cr}^{2+}: \mathrm{ZnSe}$ and $\mathrm{Fe}^{2+}: \mathrm{ZnSe}$," J. Opt. Soc. Am. B 25, 794-800 (2008).

7. U. Keller, K. J. Weingarten, F. X. Kärtner, D. Kopf, B. Braun, I. D. Jung, R. Fluck, C. Honninger, N. Matuschek, and J. A. derAu, "Semiconductor saturable absorber mirrors (SESAM's) for femtosecond to nanosecond pulse generation in solid-state lasers," IEEE J. Sel. Top. Quantum Electron. 2, 435-453 (1996).

8. S. Tsuda, W. H. Knox, S. T. Cundiff, W. Y. Jan, and J. E. Cunningham, "Mode-locking ultrafast solid-state lasers with saturable Bragg reflectors," IEEE J. Sel. Top. Quantum Electron. 2, 454-464 (1996).

9. I. Freund, "Self- $Q$-switching in ruby lasers," Appl. Phys. Lett. 12, 388-390 (1968).

10. R. J. Collins, L. O. Braun, and D. R. Dean, "A new method of giant pulsing ruby lasers,” Appl. Phys. Lett. 12, 392 (1968).
11. M. Birnbaum and C. L. Fincher, "The ruby laser: pumped by a pulsed argon ion laser," Appl. Phys. Lett. 12, 225-227 (1968).

12. A. Szabo and L. E. Erickson, "Self- $Q$-switching of ruby lasers at 77 degrees K," IEEE J. Quantum Electron. 4, 692-698 (1968).

13. H. Samelson, A. Lempicki, and V. Brophy, "Self- $Q$-switching of $\mathrm{ND}^{3+}$ SEOCL 2 liquid laser," J. Appl. Phys. 39, 4029-4030 (1968)

14. M. Birnbaum and C. L. Fincher, "Self- $q$-switched ND ${ }^{3+}-$ YAG and ruby lasers," Proc. IEEE 57, 804-805 (1969).

15. A. L. Mikaelyan, V. F. Kuprishov, Y. G. Turkov, Y. V. Andreev, and A. A. Shcherbakova, "New method for generating a giant pulse in optical generators,” J. Exp. Theor. Phys. Lett. 11, 244-246 (1970).

16. Z. Zalevsky, Y. Kapellner, I. Eyal, and N. Cohen, "Self $Q$-switching effect in a Nd:YVO ${ }_{4} / \mathrm{KTP}$ lasing unit," Opt. Eng. 45, 070506 (2006).

17. A. Szabo, "Repetitive self- $Q$-switching in a continuously pumped ruby-laser," J. Appl. Phys. 49, 533-538 (1978).

18. B. C. Weber and A. Hirth, "Efficient single-pulse emission with submicrosecond duration from a Cr:LiSAF laser," Opt. Commun. 128, 158-165 (1996).

19. B. C. Weber and A. Hirth, "Presentation of a new and simple technique of $Q$-switching with a $\operatorname{LiSrAl}_{6}: \mathrm{Cr}^{3+}$ oscillator," Opt. Commun. 149, 301-306 (1998).

20. R. S. Conroy, T. Lake, G. J. Friel, A. J. Kemp, and B. D. Sinclair, "Self- $Q$-switched $\mathrm{Nd}: \mathrm{YVO}_{4}$ microchip lasers," Opt. Lett. 23, 457-459 (1998).

21. A. V. Kir'yanov, N. N. Il'ichev, and Y. O. Barmenkov, "Excitedstate absorption as a source of nonlinear thermo-induced lensing and self- $Q$-switching in an all-fiber Erbium laser," Laser Phys. Lett. 1, 194-198 (2004).

22. S. Wolff, A. Rodionov, V. E. Sherstobitov, C. Doering, and H. Fouckhardt, "Self-pulsation in broad area lasers with transverse-mode selective feedback," Opt. Commun. 265 642-648 (2006).

23. B. N. Upadhyaya, A. Kuruvilla, U. Chakravarty, M. R. Shenoy, K. Thyagarajan, and S. M. Oak, "Effect of laser linewidth and fiber length on self-pulsing dynamics and output stabilization of single-mode Yb-doped double-clad fiber laser," Appl. Opt. 49, 2316-2325 (2010).

24. Y. Tang and J. Xu, "Effects of excited-state absorption on self-pulsing in $\mathrm{Tm}^{3+}$-doped fiber lasers," J. Opt. Soc. Am. B 27, 179-186 (2010).

25. N. Passilly, E. Haouas, V. Ménard, R. Moncorgé, and K Ait-Ameur, "Population lensing effect in Cr:LiSAF probed by Z-scan technique," Opt. Commun. 260, 703-707 (2006).

26. N. Passilly, M. Fromager, and K. Ait-Ameur, "Improvement of the self- $Q$-switching behavior of a Cr:LiSrAlF 6 laser by use of binary diffractive optics," Appl. Opt. 43, 5047-5059 (2004).

27. N. Passilly, M. Fromager, K. Ait-Ameur, R. Moncorge, J. L. Doualan, A. Hirth, and G. Quarles, "Experimental and theoretical investigation of a rapidly varying nonlinear lensing effect observed in a $\mathrm{Cr}^{3+}$ :LiSAF laser," J. Opt. Soc. Am. B 21, 531-538 (2004).

28. M. Fromager and K. A. Ameur, "Modeling of the self- $Q$-switching behavior of lasers based on chromium doped active material," Opt. Commun. 191, 305-314 (2001).

29. T. Godin, R. Moncorgé, J.-L. Doualan, M. Fromager, K Ait-Ameur, R. A. Cruz, and T. Catunda, "Optically pump-induced athermal and nonresonant refractive index changes in the reference Cr-doped laser materials: Cr:GSGG and ruby,” J. Opt. Soc. Am. B 29, 1055-1064 (2012).

30. S. A. Payne, L. L. Chase, H. W. Newkirk, L. K. Smith, and W. F. Krupke, "LiCaAlF $6 \mathrm{Cr}^{3+}$ a promising new solid-state laser material,” IEEE J. Quantum Electron. 24, 2243-2252 (1988).

31. J. J. Deyoreo, L. J. Atherton, and D. H. Roberts, "Elimination of Scattering Centers from $\mathrm{Cr}-\mathrm{LiC} C \mathrm{Al} F_{6}$, J. Cryst. Growth 113 , 691-697 (1991).

32. D. Klimm and P. Reiche, "Nonstoichiometry of the new laser host $\mathrm{LiCa \textrm {Al } F}$," Cryst. Res. Technol. 33, 409-416 (1998).

33. D. Klimm, R. Uecker, and P. Reiche, "Melting behavior and growth of colquiriite laser crystals," Cryst. Res. Technol. 40, 352-358 (2005).

34. U. Demirbas, R. Uecker, D. Klimm, and J. Wang, "A low-cost, broadly-tunable (375-433 nm \& 746-887 nm) Cr:LiCAF laser 
pumped by one single-spatial-mode diode," Appl. Opt. 51, 8440-8448 (2012)

35. J. G. Fujimoto, C. Pitris, S. A. Boppart, and M. E. Brezinski, "Optical coherence tomography: an emerging technology for biomedical imaging and optical biopsy," Neoplasia 2, 9-25 (2000).

36. U. Demirbas, M. Schmalz, B. Sumpf, G. Erbert, G. S. Petrich, L. A. Kolodziejski, J. G. Fujimoto, F. X. Kärtner, and A. Leitenstorfer, "Femtosecond Cr:LiSAF and Cr:LiCAF lasers pumped by tapered diode lasers," Opt. Express 19, 20444-20461 (2011).

37. U. Demirbas, A. Sennaroglu, F. X. Kärtner, and J. G. Fujimoto, "Comparative investigation of diode pumping for continuouswave and mode-locked $\mathrm{Cr}^{3+}$ :LiCAF lasers," J. Opt. Soc. Am. B 26, 64-79 (2009).

38. J. J. Zayhowski and C. Dill III, "Diode-pumped passively $Q$-switched picosecond microchip lasers," Opt. Lett. 19, 1427-1429 (1994).

39. B. Braun, F. X. Kärtner, U. Keller, J.-P. Meyn, and G. Huber, "Passively $Q$-switched 180 ps Nd:LaSc ${ }_{3}\left(\mathrm{BO}_{3}\right)_{4}$ microchip laser," Opt. Lett. 21, 405-407 (1996).

40. B. Braun, F. X. Kärtner, M. Moser, G. Zhang, and U. Keller, "56 ps passively $Q$-switched diode-pumped microchip laser," Opt. Lett. 22, 381-383 (1997).

41. A. Sennaroglu, Photonics and Laser Engineering: Principles, Devices, and Applications (McGraw-Hill, 2010).

42. K. J. Weingarten, B. Braun, and U. Keller, "In-situ Small-signal gain of solid-state lasers determined from relaxation oscillation frequency measurements," Opt. Lett. 19, 1140-1142 (1994).
43. A. W. Tucker, M. Birnbaum, C. L. Fincher, and L. G. DeShazer, "Continuous-wave operation of $\mathrm{Nd} \mathrm{YVO}_{4}$ at 1.06 and $1.34 \mathrm{~m}$," J. Appl. Phys. 47, 232-234 (1976).

44. M. Traiche, T. Godin, M. Fromager, R. Moncorgé, T. Catunda, E. Cagniot, and K. Ait-Ameur, "Pseudo-nonlinear and athermal lensing effects on transverse properties of $\mathrm{Cr}^{3+}$ based solid-state lasers," Opt. Commun. 284, 1975-1981 (2011).

45. T. Godin, M. Fromager, E. Cagniot, F. Porée, T. Catunda, R. Moncorgé, and K. Aït-Ameur, "Transverse pseudo-nonlinear effects measured in solid-state laser materials using a sensitive time-resolved technique," Appl. Phys. B 107, 733-740 (2012).

46. N. Passilly, M. Fromager, K. A. Ameur, R. Moncorge, J. L. Doualan, A. Hirth, and G. Quarles, "Measurement of the index-inversion coupling contributing to the time-dependent nonlinear lens effect in a $\mathrm{Cr}^{3+}$ :LiSAF laser," J. Phys. IV 119, 257-258 (2004)

47. S. M. Lima and T. Catunda, "Discrimination of resonant and nonresonant contributions to the nonlinear refraction spectroscopy of ion-doped solids," Phys. Rev. Lett. 99, 243902 (2007).

48. H. Eilers, E. Strauss, and W. M. Yen, "Photoelastic effect in $\mathrm{Ti}^{3+}$-doped sapphire," Phys. Rev. B 45, 9604-9610 (1992).

49. E. Strauss, "Bulk and local elastic relaxation around opticallyexcited centers," Phys. Rev. B 42, 1917-1921 (1990).

50. U. Demirbas, D. Li, J. R. Birge, A. Sennaroglu, G. S. Petrich, L. A. Kolodziejski, F. X. Kärtner, and J. G. Fujimoto, "Low-cost, singlemode diode-pumped Cr:Colquiriite lasers," Opt. Express 17, 14374-14388 (2009). 